\title{
Multiport Sensor RFIDs for Wireless Passive Sensing of Objects-Basic Theory and Early Results
}

\author{
Gaetano Marrocco, Member, IEEE, Lorenzo Mattioni, and Claudio Calabrese
}

\begin{abstract}
A new family of passive sensor radio-frequency identification devices is here proposed for applications in the context of wireless sensor networks. The new tags, working in the ultra-high frequency band, are able to detect the value or the change of some features of the tagged body without using any specific sensor. Such tags are provided with multiple chips embedded either within a cluster of cooperating antennas or in a single multiport antenna, and exploit the natural mismatch of the antenna input impedance caused by the change of the tagged object. A basic theory of multiport sensor tags is formulated with the purpose to describe the possible classification and detection performances in a unitary context. Some numerical examples and a first experiment corroborate the feasibility of the idea.
\end{abstract}

Index Terms-Backscattering, meander line, multiport antenna, radio-frequency identification (RFID), sensor network.

\section{INTRODUCTION}

$\mathbf{T}$ HE recent advances in wireless sensor networks (WSNs) [1]-[4] for applications in mobile and time-varying environments, are generating growing attention to low-cost and low-power wireless nodes equipped with radio and sensing ability. Such nodes are spatially distributed to ensure a cooperative monitoring of physical or application-specific conditions and parameters. Typical fields of applications for WSNs include environmental and habitat monitoring [5], [6]; disaster relief [7]; health care [8], [9]; inventory tracking and industrial processing monitoring [10]; security and military surveillance [11], [12]; and smart-spaces applications [13], [14].

The required technology improvements are related to the miniaturization of microchips and sensors by means of micromechanical technologies [15], [16] to energy savings [17] via ambient-power scavenging and energy-harvesting techniques [18], [19] and to the integration of multiple sensing devices within a single node.

Along with these technological efforts, a novel increasing trend is the integration among wireless sensor networks and radio-frequency identification (RFID) technologies. The new generation of RFID tags may include built-in sensing capabilities [20]-[22]. On the other hand, applications based on sensor networks often require means for unique identification

Manuscript received July 27, 2007; revised March 19, 2008. Published August 6, 2008 (projected).

The authors are with the Dipartimento di Informatica, Sistemi e Produzione, University of Rome "Tor Vergata," Rome 00133, Italy (e-mail: marrocco@ disp. uniroma2.it).

Color versions of one or more of the figures in this paper are available online at http://ieeexplore.ieee.org.

Digital Object Identifier 10.1109/TAP.2008.927541 and localization of individual sensors or objects. Convergence of sensing and identification technologies may thus enable a wide range of heterogeneous applications which demand tight synergy between detection and tagging.

Currently, several approaches have been proposed to provide RFID devices with enhanced sensing and detection capabilities. Active RFID tags, which make use of independent batteries or power-supply units, ensure long operating ranges and can support high data and sensor activity rates. The main drawbacks of this solution are high cost, limited lifetime, and large weight and size. Conversely, passive RFID tags are completely battery free and could be permanently embedded into tagged objects for structural, medical [23], or logistic monitoring. The major limitation of this class of system is the need of proximity to a reading device.

A significant example of an enhanced passive RFID system is given by the Wireless Identification Sensing Platform (WISP) project [24], [25], which proposed the concept of ID modulation, making use of two mercury switches and enhanced power harvesting units to mechanically switch between two commercial RFID integrated circuits (ICs).

Another prominent approach that combines passive sensing and identification is given by surface acoustic wave (SAW) technology [26], [27]. SAW devices are special microacoustic components consisting of a piezoelectric substrate, including interdigital transducers and reflecting or coupling gratings deposited on its surface. Passive SAW tags have been recently employed as sensing devices for physical or chemical parameters which are able to affect and change the propagation or reflection characteristics of the transponders.

Within this scenario, this paper proposes an investigation on a new paradigm for passive sensor RFIDs in the ultra-high-frequency (UHF) industrial-scientific-medical (ISM) band, able to detect the value or the change some tagged body's features without any a priori knowledge about the object position and orientation with respect to the querying device. The considered tags family may work without any specific embedded sensor since it exploits the dependence of the tag's input impedance and radar cross section on the physical and geometrical features of a real target. It is well experienced that when the same tag is placed onto different targets, the tag antenna's input impedance may, in some cases, undergo a mismatch and, hence, a change of the read distance can be observed. The variation of backscattered power corresponding to the impedance mismatch can be thus detected by the reader (Fig. 1). When a cluster of passive RFID tags (antenna plus IC transponder), or a single multiport tag having distinct trasponders, is deployed on the same target, several correlated backscattered signals are originated. Each of 


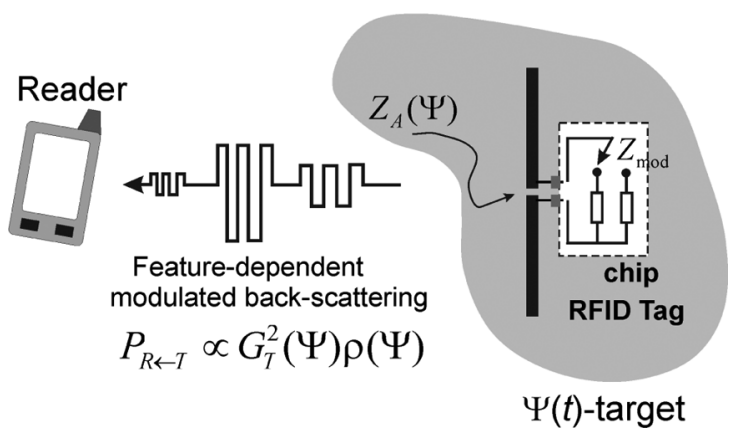

Fig. 1. Reader-tag scenario wherein the change of the target's features may produce a modulation of the backscattered power signal.

them is labeled by the unique identification code (ID) of the responding IC. These data may be available for postprocessing inside the interrogating device with the purpose of detecting the value of some target's features or their modification along with the time. The compliance of this idea with power and distance limitations of a traditional passive RFID system will be discussed here with particular attention paid to the design of multiport systems that are able to classify objects or estimate the variation of the object's features within a continuous range.

This paper introduces the leading idea of multiport tag antennas used as sensors with the help of simplified target models. Particular emphasis is devoted to introduce the overall system keypoints, to understand the physical phenomena affecting the sensing performances and to finally derive basic technical requirements for tag antenna design.

This paper is organized as follows: Section II overviews basic definitions and fundamental equations for a passive RFID system. Section III introduces the physical rationale and the adopted formalism. The multiport tag concept is then presented in Section IV, while different sensing modes and data retrieval procedures are discussed in Section V. The limitation of the sensing platform is exposed in Section VI. Finally, some numerical examples and a laboratory experiment are given in Sections VII and VIII for the very first proof of the effectiveness of the theory.

\section{BASIC DEFINITIONS OF RFID SYSTEMS}

Since the rationale of the proposed RFID platform is deeply based on the dependence of the RFID system parameters on the tagged object's features, some basic definitions are now quickly reviewed in a unitary context. At the beginning of the reader-to-tag communication protocol [29], the reader first activates the tag, placed over a target object, by sending a continuous wave which, by charging an internal capacitor, provides the required energy to perform actions. During this listening mode, the microchip exhibits an input impedance $Z_{\text {chip }}=R_{\text {chip }}+$ $j X_{\text {chip }}$, with $X_{\text {chip }}$ capacitive. The antenna impedance $Z_{A}=$ $R_{A}+j X_{A}$ has to be matched to $Z_{\text {chip }}\left(Z_{A}=Z_{\text {chip }}^{*}\right)$ for maximum power transfer. The fraction $P_{R \rightarrow T}$ of the reader input power that is absorbed by the tag is

$$
P_{R \rightarrow T}=\left(\frac{\lambda_{0}}{4 \pi d}\right)^{2} G_{R}(\theta, \phi) G_{T}(\theta, \phi) \tau P_{\mathrm{in}} \eta_{p}
$$

where $\lambda_{0}$ is the free-space wavelength, $d$ is the reader-tag distance, $G_{R}$ is the gain of the reader antenna, $G_{T}$ is the gain of the tag's antenna, placed on the target, and $\tau$ is the power transmission coefficient of the tag, defined as

$$
\tau=\frac{4 R_{\text {chip }} R_{A}}{\left|Z_{\text {chip }}+Z_{A}\right|^{2}}
$$

$\eta_{p}$ is the polarization efficiency between the reader and the tag. The tag is activated when the absorbed power exceeds the tag's microchip sensitivity threshold $p_{T}$, that is, when $P_{R \rightarrow T}>p_{T}$. Having assumed polarization-matched antennas aligned for maximum directional radiation, the longest activation distance for the tag is therefore

$$
d_{R \rightarrow T}=\max _{\theta, \phi}\left\{\frac{\lambda_{0}}{4 \pi} \sqrt{G_{R}(\theta, \phi) \hat{G}_{T}(\theta, \phi) \frac{P_{\mathrm{in}}}{p_{T}}}\right\} .
$$

The function $\hat{G}_{T}=G_{T} \tau$ is the realized gain [28] of the antenna and it is the most important performance parameter of the tag. During the next steps of the communication, the tag receives the command coming from the reader and finally sends the data back through a backscattered modulation of the continuous wave coming from the reader itself. The tag's IC acts as a programmable switching device between low and high modulating impedances $\left(Z_{\mathrm{mod}}\right)$. During the data transfer, the RFID system can be considered as a monostatic radar and, therefore, it can be characterized by the radar range equation [30] which, for the case of typical RFID tags, can be expressed as [29] and [31] in the form

$$
\frac{P_{R \leftarrow T}(d)}{P_{\text {in }}}=\left(\frac{\lambda_{0}}{4 \pi d}\right)^{4} G_{R}^{2}(\theta, \phi) G_{T}^{2}(\theta, \phi) \rho
$$

where $P_{R \leftarrow T}$ is the power received back by the reader and $\rho$ is a modulation parameter

$$
\rho=\frac{4 R_{A}^{2}}{\left|Z_{\text {mod }}+Z_{A}\right|^{2}}
$$

and it is related to the tag's radar cross section $\sigma$ according to the relation $\sigma=\lambda_{0}^{2} / 4 \pi G_{T}^{2} \rho$. The low modulation state, producing no significant backscattering $(\rho=0)$, is obtained with high impedance (virtually an open circuit $Z_{\bmod }=\infty$ ), while the high state is achieved, depending on the particular microchip family [29], either with a short circuit $\left(Z_{\text {mod }}=0\right)$ or with the listening-mode chip impedance $\left(Z_{\text {mod }}=Z_{\text {chip }}\right.$, for which $\rho=$ 1). The last case generally produces the strongest backscattering for typical UHF tags.

The maximum distance at which the backscattered power can be detected by the reader is such that this power exceeds the reader sensitivity threshold $p_{R}$. Since $p_{R} \ll p_{T}$ [32], the range of a reader-tag link is mainly constrained to the maximum tag activation distance $d_{R \rightarrow T}$, hereafter simply referred to as the link length.

\section{Physical Rationale}

Similar to any antenna immersed in or located close to a real object [33], [34], the input and radiation characteristics of a passive RFID transponder placed on a target as well as the strength 
of the backscattered power closely depend on the physical properties of the tagged object itself (e.g., on its constitutive materials, shape, temperature, humidity, or other). We denote $\Psi$ as a target's feature which could undergo changes along with the time or it has to be monitored someway. If the tag antenna has been designed for optimal performances when placed on a target with nominal $\Psi_{T}$ (e.g., such that the antenna impedance $Z_{A}\left(\Psi_{T}\right)$ in this configuration is equal to $Z_{\text {chip }}^{*}$ ), a change of one or more of the target's parameters with respect to $\Psi_{T}$ may produce a variation of the input impedance and, hence, a mismatch $\left(Z_{A} \neq Z_{\text {chip }}^{*}\right)$. Accordingly, the activation distance and the backscattered power collected at the reader port will also be modified (see Fig. 1 again). In the limiting case, the tag may be completely mismatched so that $P_{R \rightarrow T}<p_{T}$ and the tag is therefore inaccessible by the reader.

It is now useful to define the tag's activation set $\mathcal{A}(d)$ corresponding to a link length $d$, as the set of the target's (apparent) permittivity values for which the power harvested by the tag is high enough to activate it

$$
\mathcal{A}(d)=\left\{\varepsilon \mid P_{R \rightarrow T}(d, \varepsilon) \geqslant p_{T}\right\} .
$$

For the sake of clarity, let us focus on the simplified case for which the parameter to be observed is the relative real dielectric permittivity (simply permittivity $\varepsilon$ in the following text), which is supposed to be real. This case is representative for low-permittivity (dry) materials whose imaginary part of permittivity is generally much lower than the real one. Instead, such a simplification is not adequate for liquid targets having a high value of permittivity. However, the role of the target electric conductivity and, hence, the dielectric loss on the multiport tag performances, are discussed in the dedicated Section VI. The one-parameter analysis is, however, also useful for monitoring the variation of the target's shape. The change of the target's size or shape, even without modification of its constitutive material will, in fact, produce a redistribution of the reactive electric and magnetic energy stored in the antenna near field. Such an effect is macroscopically sensed by the antenna as the variation of the apparent body permittivity, in analogy to the effective dielectric constant for microstrip lines and patch antennas [35]. An experimental example in this sense will be given in Section VIII about the monitoring of the level of powder inside a container.

\section{Multiport Sensing-TAG CONCEPT}

As suggested by (4), if the reader-tag distance were known, the change in the target real permittivity could be theoretically detected by monitoring the power backscattered by the transponder.

Nevertheless, a single received data is not adequate in case of noncooperating moving objects. In fact, rather than by a real modification of the target's permittivity, a variation of the received signal could be produced by a change of the tag distance or, since the tag's radiation pattern is not isotropic, by a rotation of the target with respect to the reader. To overcome these uncertainties, the reader needs to collect multiple backscattered signals. In the proposed platform, these signals are originated from either a cluster of $N$ cooperating tags co-located onto a same target, or from a single tag provided with $N$ input ports

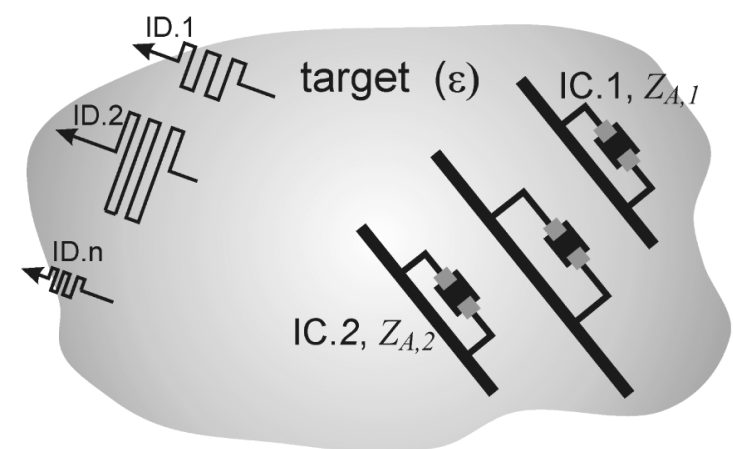

a)

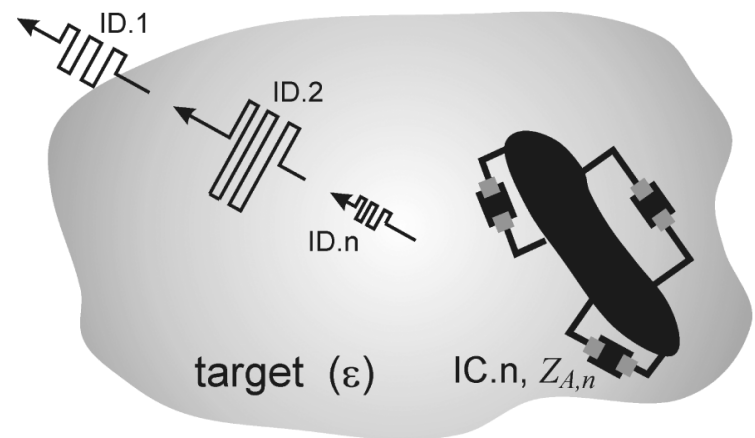

b)

Fig. 2. Multiport tag systems. (a) Cluster of colocated single-port tags (b) Single multiport tag provided with multiple chips. The ports have a unique I-D and, when the tag is placed over a target with permittivity $\varepsilon$, they exhibit different activation sets, input impedance, and a backscattering modulation parameter.

(Fig. 2), and thus with $N$ microchips, under the condition that each port has a different input impedance. In particular, we denote with $G_{T, n}$ the radiation gain of the tag when only the $n$th port is fed and the others are closed to a reference load, and with $Z_{A, n}$ the input impedance in the same condition.

Without loss of generality, all of the microchips are assumed to have the same input impedance $Z_{\text {chip,n }}=Z_{\text {chip }}$. The multiport system has to be designed so that by having fixed a target's geometry and having chosen $N$ different reference real permittivities $\left\{\varepsilon_{1}, \varepsilon_{2}, \ldots, \varepsilon_{N}\right\}$, the $n$th port impedance is matched to the microchip if the target's permittivity value is $\varepsilon_{n}$ (e.g., $\left.Z_{A, n}\left(\varepsilon_{n}\right)=Z_{\text {chip }, \mathrm{n}}^{*}\right)$. It means that, when the multichip system is placed on a particular target $\left(\varepsilon=\varepsilon_{T}\right)$, the ports will be mismatched differently $\left(Z_{A, n}\left(\varepsilon_{T}\right) \neq Z_{\text {chip }}^{*}\right)$ and, therefore, according to the discussion in the previous section, they will originate different backscattered power signals, all of them carrying information about the unique target's permittivity.

The resulting overall device is a multiport sensor RFID (S-RFID) tag which, as shown later on, adds specific sensing capabilities to the typical identification features and employs the same fabrication technology of the conventional RFID tags, since the extra cost is simply given by the need of multiple microchips. Each port of the S-RFID tag is then associated by the following data:

- $I D_{n}$ : the identification code of the $n$th port microchip;

- $\varepsilon_{n}$ : the reference target's permittivity for which the port is matched to the microchip; 
- $\mathcal{A}_{n}(d)$ : the port activation set defined as in (6);

- $d_{n}(\varepsilon)$ : the maximum activation distance of the $n$th chip;

- $\tau_{n}$ : the power transmission coefficient toward the $n$th chip;

- $\rho_{n}(\varepsilon)$ : the backscattering modulation parameter.

\section{Sensing of Target's Permittivity}

Depending on the link length $d$ and on the particular design of the multiport S-RFID tag, either multiple ICs will respond (overlapping of activation sets) and, hence, the reader will be able to collect multiple backscattered signals, or only a port will be, at most, activated and the reader will receive a single ID. Two different sensing modes can be correspondingly achieved: analog sensing (multiple responding ICs) and discrete sensing (single responding IC). For both the cases, it is useful to introduce the sensing range $\mathcal{S}(d)$ of the multiport S-RFID tag, as the set of all possible values of the target's permittivity which could be detected, in some way, at a distance $d$. As discussed next, the span of the multiport S-RFID range is controlled by the choice of the reference sequence $\left\{\varepsilon_{n}\right\}$ involved in the tag design.

\section{A. Analog Sensing and Retrieval Procedure}

Overlapped activation sets $\mathcal{A}_{n}$ for the multiport system can be achieved if the multiport tag is designed for close reference permittivities $\left\{\varepsilon_{n}\right\}$. In a real application, the port will therefore exhibit similar (but not identical) power transmission coefficients $\tau_{n}$ so that multiple microchips will be turned on. For any couplet of backscattered signals received by the reader, each with a different modulation parameter $\rho_{n}(\varepsilon)$, the unknown reader-tag distance is dropped by calculating the backscattering power ratio $p_{i j}$ between the received powers in (4)

$$
p_{i j}(\theta, \phi, \varepsilon)=\frac{P_{R \leftarrow T, i}}{P_{R \leftarrow T, j}}=\left[\frac{G_{T, i}(\theta, \phi, \varepsilon)}{G_{T, j}(\theta, \phi, \varepsilon)}\right]^{2} \frac{\rho_{i}(\varepsilon)}{\rho_{j}(\varepsilon)} .
$$

However, $p_{i j}$ is still affected by the uncertainty on the tag orientation $(\theta, \phi)$ with respect to the reader. It is therefore required that the multiport tag design satisfy the additional condition of proportional gain patterns, for example

$$
\frac{G_{T, i}(\theta, \phi, \varepsilon)}{G_{T, j}(\theta, \phi, \varepsilon)}=f(\varepsilon)
$$

As discussed in the example section, this condition could be roughly achieved by considering a cluster of two antennas having a similar geometry and placed at a proper distance.

The retrieval procedure is now described by means of an example involving a two-port system able to backscatter two different IDs toward the reader. An overlapping configuration between the activation ranges is illustrated in Fig. 3(a). When the $I D_{1}$ and the $I D_{2}$ are received by the reader, the unknown target dielectric permittivity $\varepsilon_{T}$ will belong to the intersection of the two activation regions, e.g.,

$$
\varepsilon_{T} \in\left[\mathcal{A}_{1} \cap \mathcal{A}_{2}\right]
$$

and, therefore, the $p_{12}$ ratio can be calculated as in (7). The value of the target's permittivity is hence retrieved by using a calibration curve $\varepsilon\left(p_{12}\right)$ which associates an unknown permittivity value of the target [Fig. 3(b)] to the actual backscattered

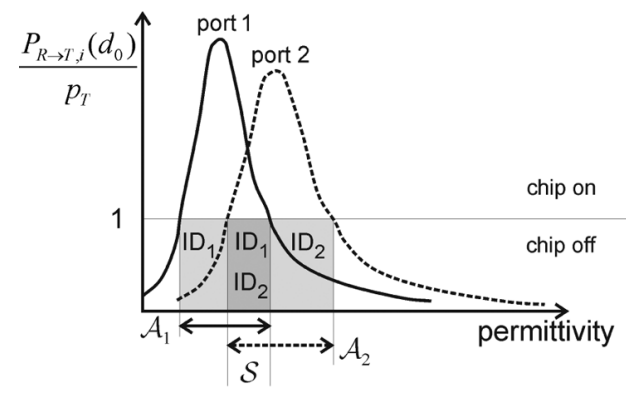

a)

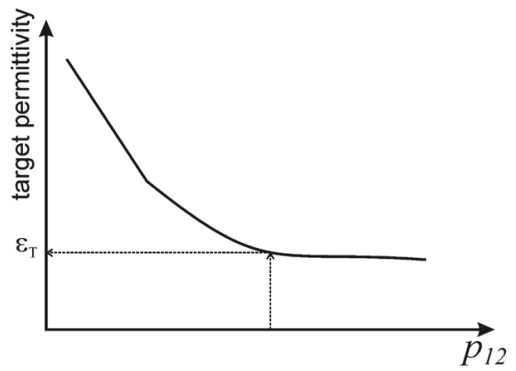

b)

Fig. 3. (a) Typical activation sets and sensing range of a two-port RFID tag, designed to work in analog-sensing mode. Up to two IDs can be transmitted back to the reader depending on the target's permittivity and distance. (b) Example of calibration curve $\varepsilon\left(p_{12}\right)$ associating the measured backscattered power ratio with a target's permittivity value.

power ratio, measured by the reader. The application of such a technique therefore requires a preliminary analysis to produce calibration curves for the specific class of objects by means of measurements or numerical simulations on simplified or wellrepresented target models through a sweep of the parameter under observation and calculating the resulting backscattered power ratio. The so obtained database, together with the retrieval procedure, have to be embedded in the reader's (post)processing unit.

The retrieval procedure can be extended to a larger number of ports but in such a case, more advanced inversion techniques need to be developed.

The multiport S-RFID can be therefore used as an analog estimator, theoretically able to retrieve information about the target's permittivity value inside the permittivity range for which at least two microchips are activated. The global S-RFID range $\mathcal{S}(d)$ is given by the merging of the activation sets shared by couplets of ports

$$
\mathcal{S}(d)=\bigcup_{m, n=1, \ldots, N ; m \neq n}\left\{\mathcal{A}_{m}(d) \bigcap \mathcal{A}_{n}(d)\right\} .
$$

The resolution in the permittivity retrieval depends on the sensibility of the gain ratio in (8) to the reader-tag orientation. With reference to Fig. 4, an approximate satisfaction of (8) will introduce an uncertainty in the $\varepsilon \leftrightarrow p_{i j}$ functional relationship since, for a given target permittivity, the received backscattering power ratio could be any value in the following range, depending on the reader-tag orientation:

$$
p_{i j, \min }(\varepsilon)<p_{i j}(\varepsilon) \leq p_{i j, \max }(\varepsilon)
$$

where $p_{i j, \min / \max }(\varepsilon)$ are the curves corresponding, for each $\varepsilon$, to the minimum and maximum-over angle power ratio (e.g., 


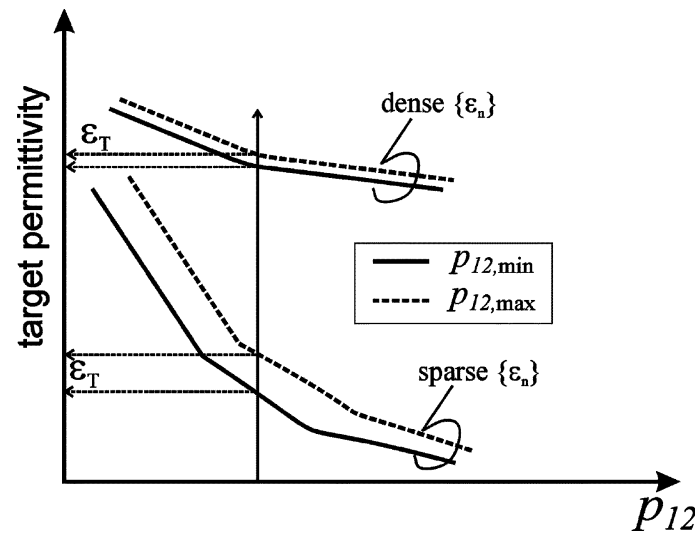

Fig. 4. Uncertainty over the reader-tag orientation may introduce a finite resolution in the permittivity retrieval which is better for low-slope power ratios corresponding to multiport tags designed for a dense set of reference permittivities $\left\{\varepsilon_{n}\right\}$.

$\left.p_{i j, \min / \max }(\varepsilon)=\min / \max \left[p_{i j},(\theta, \phi, \varepsilon)\right]\right)$. Consequently, when a $p_{i j}$ is collected by the reader, the unknown target permittivity given by the calibration curve will belong to the uncertainty range

$$
\varepsilon\left(p_{i j, \min }\right) \leq \varepsilon_{T} \leq \varepsilon\left(p_{i j, \max }\right)
$$

and, therefore, the maximum resolution achievable, in absence of other noise or other instrumental errors, is

$$
\Delta \varepsilon_{T}=\frac{1}{2}\left|\varepsilon\left(p_{i j, \max }\right)-\varepsilon\left(p_{i j, \min }\right)\right| .
$$

Another factor which plays an important role in the retrieval resolution is the slope of the calibration curves $\varepsilon\left(p_{i j}\right)$. Grazing slopes (see Fig. 4) are produced by a dense set of reference permittivities $\left\{\varepsilon_{n}\right\}$ since these will provide backscattering signals of comparable strength. This configuration leads to high resolution (small $\Delta \varepsilon_{T}$ ) over a narrow sensing range (e.g., small variation of the permittivity may be detected by means of a large dynamic of $p_{i j}$ ). On the converse, a high slope is produced by a sparse set of reference target's permittivities which give sensibly different ports' matching and more different backscattered powers. In this case, a larger span of the parameter space is achieved at the expense of reduced resolution.

The design choice of $\left\{\varepsilon_{n}\right\}$ is therefore suggested by the span of the dielectric change to be monitored at the required resolution.

\section{B. Discrete Sensing and Classification}

To classify objects according to their (apparent) permittivity belonging to a discrete set $\left\{\varepsilon_{1}, \varepsilon_{2}, \ldots, \varepsilon_{N}\right\}$, the multiport tag has to be designed using the previous permittivities as the reference set, under the condition that the corresponding activation sets are disjoined (Fig. 5), For example

$$
\bigcap_{n=1, \ldots, N} A_{n}(d)=0 \text {. }
$$

Accordingly, in case the multiport tag will be placed over any of the $\varepsilon_{k}$ targets, only the $k$ th chip will transmit back its own $I D_{k}$, so classifying the target feature. An ID modulation [36] is hence established by means of a classification table, including

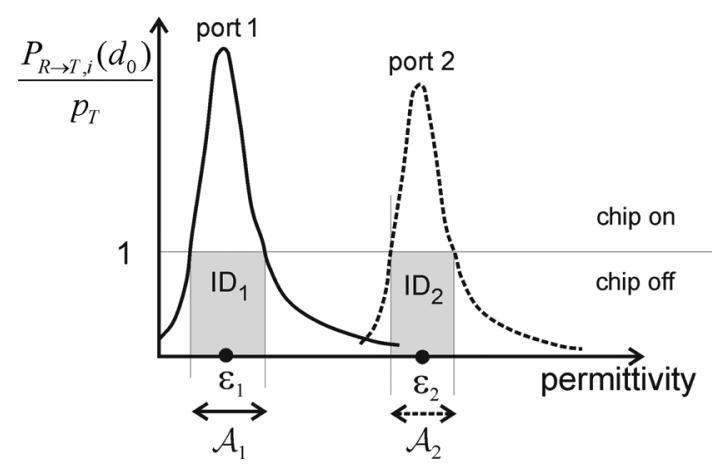

Fig. 5. Activation sets for a two-port tag designed for discrete sensing.

\begin{tabular}{|c|c|c|c|c|c|}
\hline target $\varepsilon_{2}$ & $\begin{array}{l}\mathrm{ID}_{1} \\
\mathrm{ID}_{2}\end{array}$ & $\mathrm{ID}_{2}$ & $\mathrm{ID}_{2}$ & $\mathrm{ID}_{2}$ & / \\
\hline target $\varepsilon_{1}$ & $\begin{array}{l}\mathrm{ID}_{1} \\
\mathrm{ID}_{2}\end{array}$ & $\begin{array}{l}\mathrm{ID}_{1} \\
\mathrm{ID}_{2}\end{array}$ & $\mathrm{ID}_{1}$ & / & I \\
\hline
\end{tabular}

TABLE I

ClassificATION OF A TwO-PORT TAG

the correspondences $I D_{k} \leftrightarrow \varepsilon_{k}$. In this case, the S-RFID range is trivially a discrete set

$$
\mathcal{S}(d)=\left\{\varepsilon_{1}, \ldots, \varepsilon_{N}\right\}
$$

Discrete sensing requires high selectivity (see again Fig. 4) of the tag performances, for example

$$
\hat{G}_{T, n}\left(\varepsilon_{m}\right) \rightarrow 0 \mathrm{iff} \varepsilon_{m} \neq \varepsilon_{n}
$$

which, according to (3), means that the read distance of each chip, when the tag is placed onto a target with permittivity $\varepsilon_{m}$ that differs from the port's own reference permittivity $\varepsilon_{n}$, should be very small.

The design of the multichip tag antenna will therefore pursue the maximization of $\hat{G}_{T, n}\left(\varepsilon_{n}\right)$ for large operational distance and the condition in (16) for classification selectivity. It is useful to recall that as shown in [23], the use of high impedance-phase-angle microchips will produce tags that are matched over a smaller band, in comparison with low impedance-phase-angle ones. Therefore, the selectivity of the multiport tags in discrete mode will benefit from the use of microchips of the first type.

In case condition (16) was not satisfied and, hence, multiple IDs could be emitted by the tag, the target's classification could be still possible provided that the classification table associates unique sets of IDs to a specific target to be recognized. To explain this concept, a two-port tag is now considered, with reference permittivities $\left\{\varepsilon_{1}, \varepsilon_{2}\right\}$. Two remarkable read distances can be related to the $j$ th port (e.g., $\left.d_{j}\left(\varepsilon_{i}\right), i=1,2\right)$ in case the tag were placed over a $\varepsilon_{1}$ or $\varepsilon_{2}$ target, respectively. Assuming, for instance, that the resulting four read distances are ranked as

$$
d_{1}\left(\varepsilon_{2}\right)<d_{2}\left(\varepsilon_{1}\right)<d_{1}\left(\varepsilon_{1}\right)<d_{2}\left(\varepsilon_{2}\right)
$$

the emitted IDs can be represented into a classification table (Table I) which gives the emitted IDs from the multitag system depending on the tagged objects and on the reader-tag distance. 


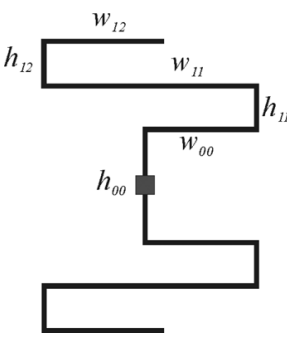

Fig. 6. Scheme for nonuniform symmetric meander line antenna (MLA) with an indication of parameters to be optimized.

The table is filled according to the following rule: for any cell along the row of the target $\varepsilon_{m}$, the $I D_{n}$ will be present if $d_{n}\left(\varepsilon_{m}\right)$ is right apart from the considered cell.

For a reader-tag distance within $d_{1}\left(\varepsilon_{2}\right)<d<d_{2}\left(\varepsilon_{2}\right)$, the target $\varepsilon_{2}$ is recognized if the reader has received the only $I D_{2}$ code, while the target $\varepsilon_{1}$ is correctly classified in the range $d_{1}\left(\varepsilon_{2}\right)<d<d_{1}\left(\varepsilon_{1}\right)$ if at least the $I D_{1}$ code has been collected. At a distance $d<d_{1}\left(\varepsilon_{2}\right)$, the two microchips are always on, for both the target's permittivities and, hence, it is not possible to achieve any classification.

Many other alternative configurations may be possible, depending on the ranking of $\left\{d_{j}\left(\varepsilon_{i}\right)\right\}$. A different case, when one of the objects to be classified is a lossy liquid, will be discussed in the example section.

From the antenna point of view, the design effort is to achieve the proper effective gain at the tag's ports so that the operational read distance could suite the specific application's requirements.

\section{ON THE SENSING OF Lossy OBJects}

Any dielectric material generally exhibits an equivalent complex dielectric permittivity $\dot{\varepsilon}=\varepsilon_{\mathrm{Re}}-j \varepsilon_{\mathrm{Im}}$ where the imaginary part accounts for losses by ohmic conduction and dielectric hysteresis [37]. The loss of the target where the tag is placed on could sensibly affect the performance or even the feasibility of the proposed multiport sensing tag. High losses mean a reduced gain of the tag and a small antenna quality factor $Q$ [38]. In the RFID context, the presence of a highly lossy target is such that the tag's electrical performances (impedance, gain, and other secondary quantities, such as the effective gain and the radar cross section) are not very sensitive to the variations of the target's complex permittivity in the surroundings of a central value. This concept is here clarified by the help of a sample concerning meander-line antenna (MLA) tags (Fig. 6) over a lossy homogeneous half-space. The MLA tag has been recently investigated [39] and such a layout offers many degrees of freedom to match different kinds of microchips and target's materials.

Having fixed the complex permittivity of the half space target, the microchip impedance, and the maximum size of the antenna, the $M$ vertical and horizontal segments of the MLA $\left\{h_{m k}, w_{m k}\right\}, m=1,2$, and $k=1,2$ are optimized by a genetic algorithm (GA) [40] procedure aimed at maximizing the following fitness function:

$$
F=G_{T} \tau
$$

TABLE II

MLA TAG SIZES

\begin{tabular}{|c|c|c|}
\hline$[\mathrm{mm}]$ & MLA for $\dot{\varepsilon}^{\text {high }}$ & MLA for $\dot{\varepsilon}^{\text {low }}$ \\
\hline$h_{00}$ & 3.2 & 7.0 \\
\hline$h_{11}$ & 2.7 & 14.0 \\
\hline$h_{12}$ & 0.6 & 2.5 \\
\hline$w_{00}$ & 20.0 & 11.6 \\
\hline$w_{11}$ & 9.4 & 27.4 \\
\hline$w_{12}$ & 9.4 & 40.0 \\
\hline
\end{tabular}

The GA is coupled with a moment method solver [41] to predict the electromagnetic performances of each antenna of the GA population. The dielectric half space is accounted for by means of the Sommerfeld integrals.

Two different single-turn MLA antennas have been independently optimized at $f=870 \mathrm{MHz}$, respectively, for usage over low-permittivitty low-loss material $\left(\dot{\varepsilon}^{\text {low }}=3-j 0.03, \tan \delta=\right.$ $10^{-2}$ ), resembling a dry object or a type of oil, and a high-permittivity high-loss liquid $\left(\dot{\varepsilon}^{\text {high }}=70-j 7, \tan \delta=10^{-1}\right)$ ), resembling water or other beverages [42]. The resulting size of the antenna layout, able to match a microchip impedance $Z_{\text {chip }}=50-j 200 \Omega$, is reported in Table II. The overall sizes of the MLA for low- and high-permittivity half-spaces are $4 \mathrm{~cm}$ $\times 4 \mathrm{~cm}$ and $1 \mathrm{~cm} \times 1 \mathrm{~cm}$, respectively. In both layouts, the conductor trace width is set to $0.5 \mathrm{~mm}$.

The variation of the $G_{T}^{2} \rho$ function, which is proportional through (4) to the backscattered power from the tag to the reader $\left(P_{R \leftarrow T}\right)$, is calculated for both antennas by the moment method when the target complex permittivity is continuously swept in the ranges

$$
\begin{aligned}
& \left|\varepsilon_{\mathrm{Re}}-\varepsilon_{\mathrm{Re}}^{\text {nom }}\right|<\Delta \varepsilon_{\mathrm{Re}} \\
& \left|\varepsilon_{\mathrm{Im}}-\varepsilon_{\mathrm{Im}}^{\text {nom }}\right|<\Delta \varepsilon_{\mathrm{Im}}
\end{aligned}
$$

where $\dot{\varepsilon}^{\text {nom }}=\varepsilon_{\mathrm{Re}}^{\text {nom }}-j \varepsilon_{\mathrm{Im}}^{\text {nom }}$ is the nominal permittivity of the target for which the tag is specifically optimized $\left(\dot{\varepsilon}^{\text {nom }}=\left\{\dot{\varepsilon}^{\text {high }}, \dot{\varepsilon}^{\text {low }}\right\}\right)$, and $\Delta \varepsilon_{\operatorname{Im}}=\tan \delta \Delta \varepsilon_{\text {Re }}$. For each value of the complex permittivity, the variation $\Delta\left[G_{T}^{2} \rho\right](\dot{\varepsilon})=\left[G_{T}^{2} \rho\right](\dot{\varepsilon}) /\left[G_{T}^{2} \rho\right]\left(\dot{\varepsilon}^{\text {nom }}\right)$ (in decibels) is reported in Fig. 7. It is expected that in order to implement the multiport sensing tag concept, such a function should exhibit a significant sensitivity to the change in the target complex permittivity. From the figure, is clear that the $G_{T}^{2} \rho$ is mainly affected by the change of the real part of the permittivity, especially for the case of low-permittivity targets for which a small change of $\varepsilon_{\text {Re }}$ (few units) produces a variation of 10 $\mathrm{dB}$, or even more, in the received backscattered signal. In the case of high-permittivity (liquid-like) materials instead, the same amount of permittivity variation gives only a few decibel scattered power changes due to the more evident smoothing effect of losses than in the previous case. It is therefore clear that the analog sensing-mode concept applies better to low-loss materials while it only permits to detect large changes of the target in case of high-loss dielectrics. Low-loss materials generally have low permittivity and poor water content. However, the discrete sensing and classification among low-permittivity and high-permittivity objects could be still feasible as shown in the example section. 


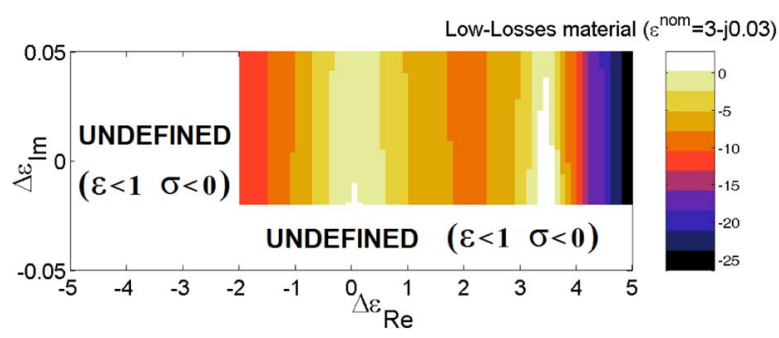

a)

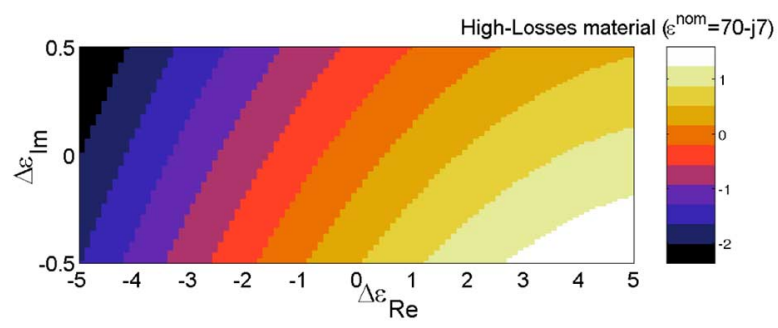

b)

Fig. 7. Sensibility of a meander-line tag performances, at $870 \mathrm{MHz}$, to the variation of the dielectrical properties of two different targets. (a) Low-permittivity target $\left(\dot{\varepsilon}^{\text {low }}=3-j 0.03\right)$. (b) High-permittivity target $\left(\dot{\varepsilon}^{\text {high }}=70-j 7\right)$. The decibel gray scale gives the $G_{T}^{2} \rho$ variation of two different tags, respectively optimized for $\dot{\varepsilon}^{\text {low }}$ and $\dot{\varepsilon}^{\text {high }}$ homogeneous half-spaces (sizes in Table I), when the physical parameters are swept around the nominal values $\dot{\varepsilon}^{\text {low }}$ and हैhigh.

\section{NUMERICAL EXAMPLES}

All of the significant performance parameters previously introduced are now numerically discussed throughout some tag layouts operating in analog and discrete sensing modes involving a cluster of two MLA antennas.

The aim is mainly to prove the feasibility of the basic idea of the multiport S-RFID tag with some degrees of generality, rather than to consider a true antenna design problem and, hence, some simplifications are introduced in the computational electromagnetic models. A canonical reference geometry is assumed for the target, consisting of a homogeneous or layered dielectric slab, orthogonal to the $z$-axis and bounded within $-h<z<0$. The tag is placed at $z=0$. Configurations with $h \rightarrow \infty$ (half-space) can model large and deep targets, such as walls or containers. Intermediate thickness could instead account for the tag response over finite objects, such as glass, liquid-filled containers, or sacs. These strong simplifications aim to speed up the tag antenna optimization by GA since the electromagnetic modeling by the moment method, requires evaluating each antenna of the population and can simply account for the target by the Sommerfeld integrals, avoiding a full mesh of complicated objects. The tag design for real applications, however, should really include a more detailed representation of the target. This issue is beyond the scope of this paper, even if a first example is given in the next experimental section.

The working frequency is $870 \mathrm{MHz}$ in both examples and the backscattered power is calculated by considering the modulation parameter $\rho$ in (5) for the case $Z_{\text {mod }}=Z_{\text {chip }}$. No hypothesis about the reader's geometry and polarization is done while its performances are only accounted through the emitted EIRP that is equal to $3.2 \mathrm{~W}$, which corresponds to the maximum permitted strength by European regulations [43].

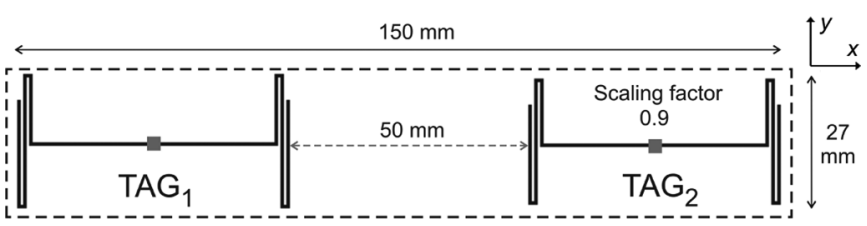

Fig. 8. Layouts of the two MLA tags placed over a homogeneous half-space target and matched to a microchip having input impedance $Z_{\text {chip }}=50-$ $j 200 \Omega$. The tags have been optimized for the target's permittivities $\varepsilon=\{3,4\}$. Dimensions expressed in millimeters for $\mathrm{MLA}_{1}$, according to the scheme in Fig. $6: h_{00}=50, h_{11}=h_{12}=0.5, w_{00}=13.7, w_{11}=26.9, w_{12}=21.62$. $\mathrm{MLA}_{2}$ is a scaled reduced replica of MLA 1 (scaling factor: 0.9).
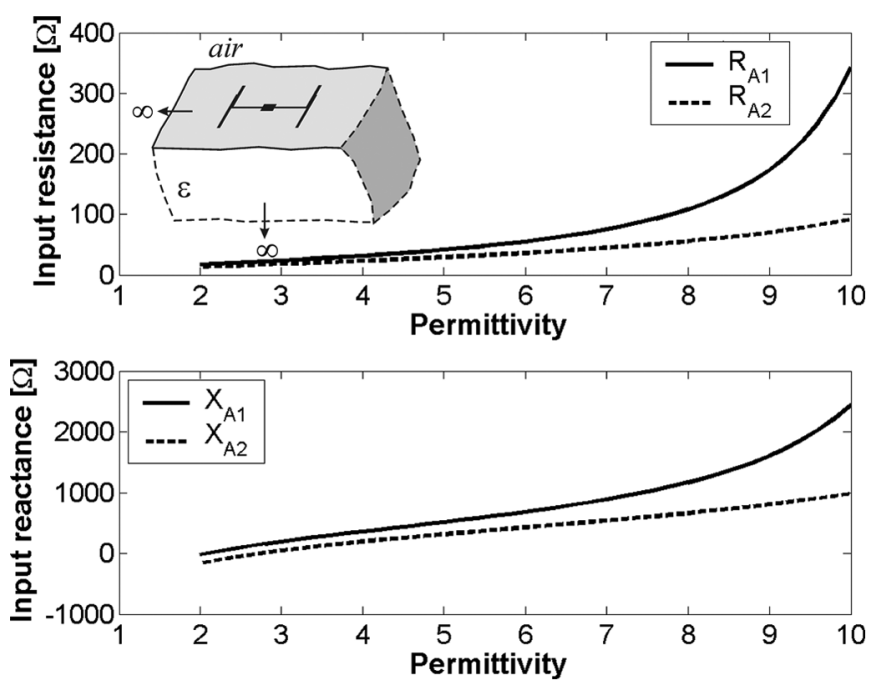

Fig. 9. Sensing of lossless material: Variation of the input impedances, at 870 $\mathrm{MHz}$, of the two antennas with respect to the dielectric permittivity of the homogeneous half-space target. The two antennas have been designed so that they exhibit similar input impedances in correspondence of the different target's permittivities.

\section{A. Permittivity Analog Sensing of Lossless Targets}

A two-port cluster of meander line antennas is here considered for the analog sensing of the permittivity of a lossless homogeneous half-space, approximately in the range $3<\varepsilon<4$. The two MLAs have been independently designed to match a microchip impedance $Z_{\text {chip }}=50-j 200 \Omega$ (sensitivity $p_{T}=$ $10 \mu \mathrm{W}$ ), having constrained the size of each one within a $3 \mathrm{~cm}$ $\times 5$-cm rectangle. The reference permittivities are $\varepsilon_{1,2}=\{3,4\}$ and the first MLA was optimized, again by the GA procedure in [39], to maximize the realized gain in case of $\varepsilon_{1}=3$. The second MLA is a scaled replica of the first one, with the scaling factor (0.9) chosen so that $Z_{A, 2}\left(\varepsilon_{2}\right) \simeq Z_{\text {chip }}^{*}$. In this case, the two MLA (Fig. 8) will have a similar gain pattern, as required to achieve a simple data inversion as in (12).

Further electromagnetic computations, also involving the moment method, are then performed to characterize the behavior of the whole two-MLA tag, including interport coupling, with the two antennas placed along the E-plane at a distance of $5 \mathrm{~cm}$, as in Fig. 8. The variations of the impedance, with respect to the change of the permittivity of the canonical target model (see the curves in Fig. 9), are computed for each tag when the other is in open circuit. The interantenna coupling is correctly taken 


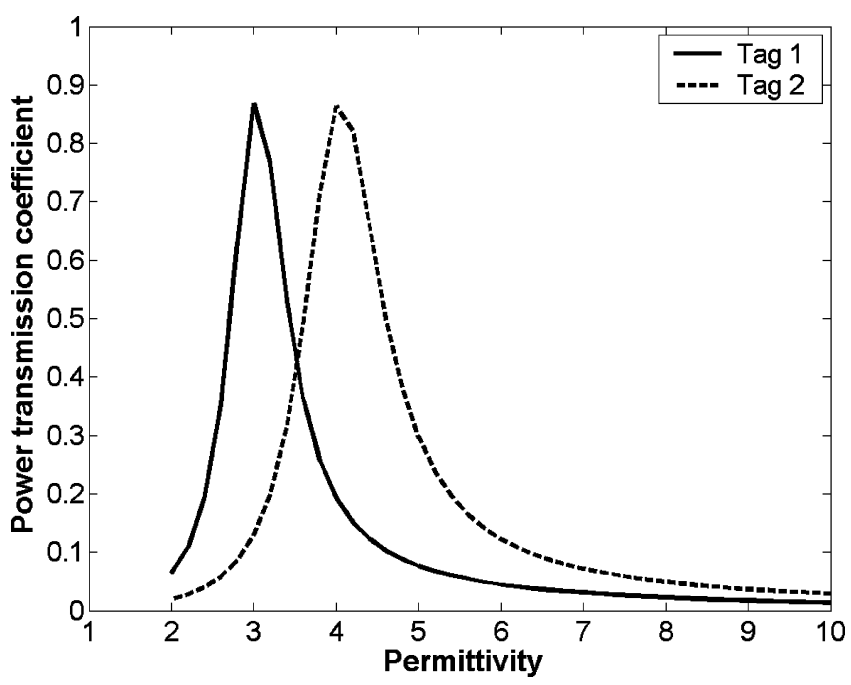

Fig. 10. Sensing of lossless materials: power transmission coefficients $\tau_{1}$ and $\tau_{2}$ of the two-port cluster with respect to the dielectric variation of the target.

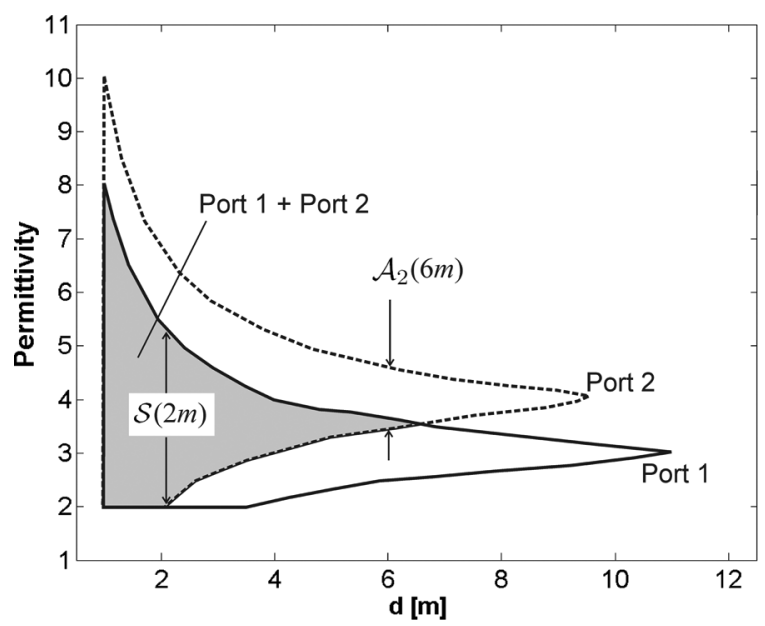

Fig. 11. Sensing of lossless materials: Activation sets $\mathcal{A}_{n}(d)$ for the two ports. The sensing range $\mathcal{S}(d)$ of the multiport S-RFID tag is given by the overlapping shadowed region.

into account by the moment method modeling. The power transmission coefficients $\tau_{1}$ and $\tau_{2}$ are nearly exact-shifted replicas (Fig. 10) as expected since the two antennas are of similar shape.

The multiport activation sets $\mathcal{A}_{n}(d)$ and the S-RFID ranges $\mathcal{S}(d)$, defined in (6) and (10), respectively, are graphically rendered within a permittivity-distance diagram (Fig. 11). For any reader-tag distance $d$, the activation set of the $n$th port spans between the upper and lower curves and has the same line style and, as expected, $\mathcal{A}_{n}$ reduces as the reader-tag distance increases. The overlapping (shadowed) region gives the S-RFID range $\mathcal{S}(d)$, wherein both the microchips are activated and, therefore, the reader is able to collect two different signals. For a reader-tag distance greater than $6 \mathrm{~m}$, the S-RFID range vanishes and only a single port can be activated, depending on the target permittivity. In this case, the S-RFID system can only work as a classifier (e.g., able to distinguish $(\varepsilon=3)$ targets from $(\varepsilon=4)$ targets $)$.

Having chosen antennas with similar shapes, the variation of the ratio between the tags' radiation patterns in the air half space
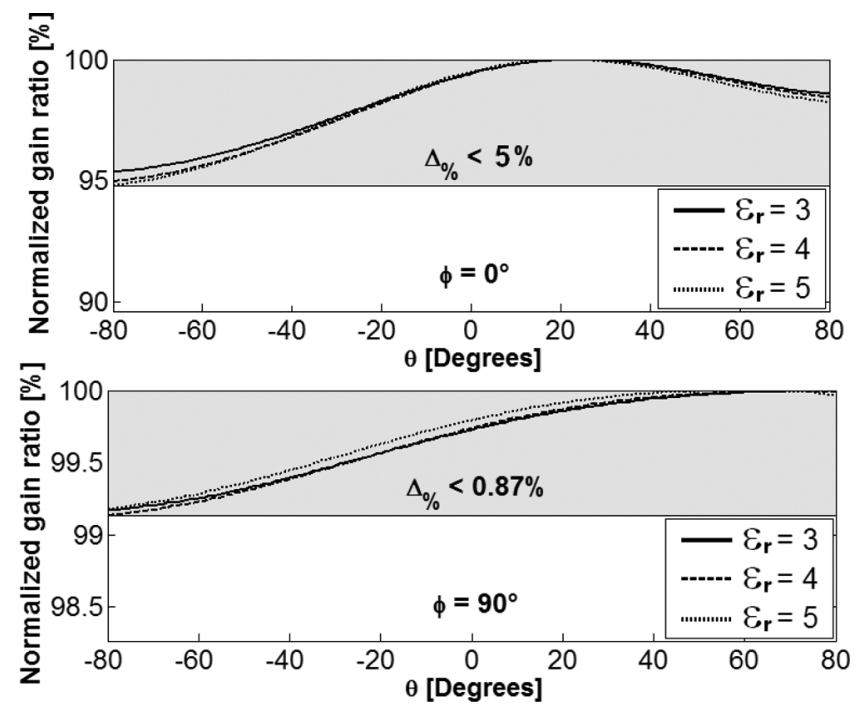

Fig. 12. Sensing of lossless materials: normalized ratio $\left(G_{T, 1} / G_{T, 2}\right)^{2}$ along the principal planes for the two-MLA tag of Fig. 8 for some values of the halfspace permittivity. The MoM calculation includes the interantenna coupling (e.g., the considered patterns are the embedded gain pattern of each tag in presence of the other one) (in open-circuit configuration).

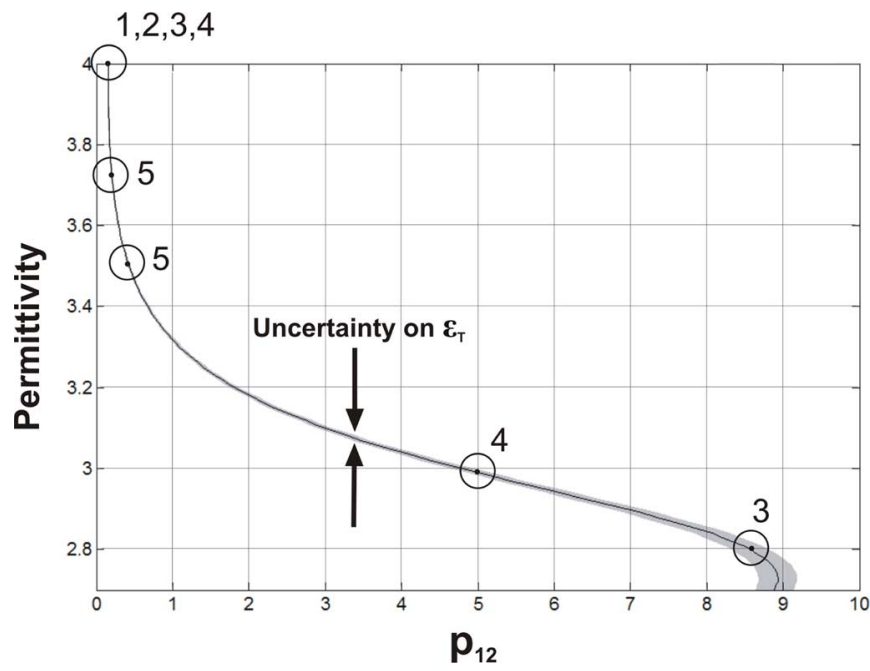

Fig. 13. Sensing of lossless materials: $\varepsilon_{T} \leftrightarrow p_{12}$ relationship with indication of the uncertainty in permittivity retrieval due to the angular dependence of the gain ratio as in Fig. 12. Markers bound the meaningful portions of the curves at the indicated reader-tag distances (in meters). The continuous line indicates the ideal $\varepsilon \leftrightarrow p_{i j}$ relationship in case of exactly similar gain patterns (e.g., $p_{i j}$ is angle independent).

$\left[G_{T, 1}(\theta, \phi) / G_{T, 2}(\theta, \phi)\right]^{2}$ is less than $5 \%$ (Fig. 12). As discussed in Section V [see (12) and (13)], such an angular variation will produce an uncertainty over the permittivity retrieval which, as visible in the $\varepsilon\left(p_{12}\right)$ chart of Fig. 13, is theoretically less than $\varepsilon_{T} \pm 0.05$, at lower values of tag's permittivity and even less for higher values of $\varepsilon_{T}$. The meaningful portion of the curve actually depends on the reader-tag distance and can be deduced from the S-RFID range in Fig. 11. Such a portion is bounded, in Fig. 13, by a couplet of distance markers. For instance, the system is able to detect a continuous target's dielectric variation in the range $3<\varepsilon_{T}<4$ up to a distance $d=4 \mathrm{~m}$, while even larger parameter dynamics $\left(2.8<\varepsilon_{T}<4\right)$ can be captured by the multiport tag in case the read distance was $d=3 \mathrm{~m}$. 


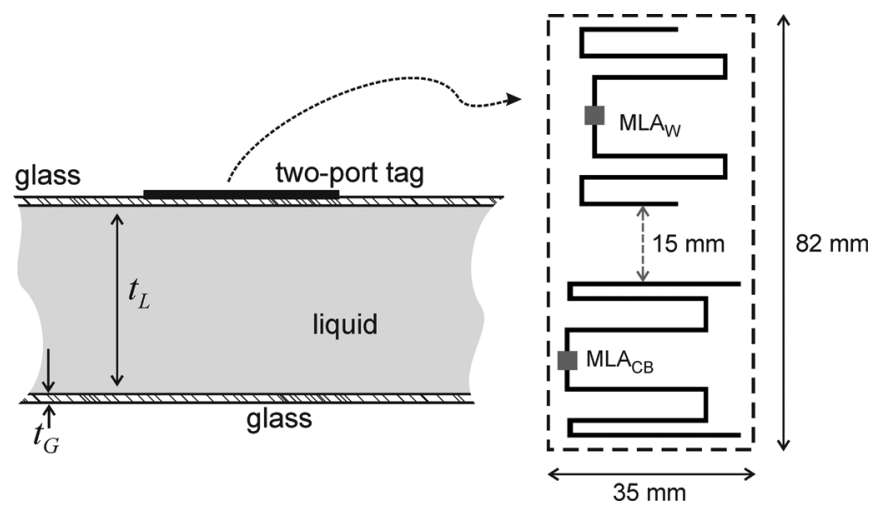

Fig. 14. Classification of liquids: two-MLA tag system over a layered medium resembling a glass-bottle filled with a liquid (layers' thickness $t_{G}=3 \mathrm{~mm}$ and $t_{L}=8 \mathrm{~cm}$. Antenna size (in millimeters) according to the layout in Fig. 6 $\mathrm{MLA}_{W}=\left\{h_{00}=16.2, h_{11}=4.3, h_{12}=5.5, w_{00}=21.8, w_{11}=\right.$ $\left.19.6, w_{12}=18.4\right\} ; \mathrm{MLA}_{\mathrm{CB}}=\left\{h_{00}=12.2, h_{11}=6.5, h_{12}=\right.$ $\left.3.0, w_{00}=28.5, w_{11}=27.9, w_{12}=35.1\right\}$. MLA trace width: $0.5 \mathrm{~mm}$.

TABLE III

PERFormances of THE Two-Port TAG FOR LiQuid Classification

\begin{tabular}{|c|c|c|}
\hline & water & chlorobenzene \\
\hline \multirow{3}{*}{ MLA $_{W}$} & $\tau=0.73$ & $\tau=0.07$ \\
& $G_{T} \tau=-17.1 d B$ & $G_{T} \tau=-16.3 d B$ \\
& $d_{W}\left(\dot{\varepsilon}_{W}\right)=2.2 m$ & $d_{W}\left(\dot{\varepsilon}_{C B}\right)=2.5 m$ \\
\hline \multirow{3}{*}{$\operatorname{MLA}_{C B}$} & $\tau=0.02$ & $\tau=0.1$ \\
& $G_{T} \tau=-32.6 d B$ & $G_{T} \tau=-14.9 d B$ \\
& $d_{C B}\left(\dot{\varepsilon}_{W}\right)=0.4$ & $d_{C B}\left(\dot{\varepsilon}_{C B}\right)=3.4 m$ \\
\hline
\end{tabular}

\section{B. Classification of Liquid Targets}

This example permits describing the multiport sensing mode for the classification of two liquid targets having the same appearance but rather different permittivity, such as the water $\left(\dot{\varepsilon}_{W}=80-j 7\right)$ and the chlorobenzene $\left(\dot{\varepsilon}_{\mathrm{CB}}=5.61-j 0.2\right)$ which is a flammable and highly toxic compound [44]. A two-port tag (cluster) is designed, as was done previously, by the integration of two MLA antennas matched to a high-impedance phase-angle microchip $\left(Z_{\text {chip }}=15-j 450 \Omega\right.$, sensitivity $p_{T}=10 \mu \mathrm{W}$ ) to improve the selectivity of the classification, as discussed in Section V-B. The target model is now a semifinite geometry (see Fig. 14 left) (e.g., a three-layer medium) where a $t_{L}=8-\mathrm{cm}$ liquid slab is sandwiched between two $t_{G}=3-\mathrm{mm}$ glass layers. This geometry is a model of a filled bottle, rough enough to simplify the application of the GA optimization. Actually, the layered medium can be still accounted for by the moment method through the Sommerfeld Green function, making the MLA optimization fast.

A first tag $\left(\mathrm{MLA}_{W}\right)$ is optimized with respect to $G_{T} \tau$ for the water target (Fig. 14, right) and its performances have been calculated also when it is placed over the chlorobenzene target. From the first row of Table III, it can be observed that although the $\mathrm{MLA}_{W}$ over chlorobenzene is nearly completely mismatched $(\tau=0.07)$, the much lower losses than in the case of water (for which $\tau=0.73$ ) make the two read distances $d_{W}\left(\dot{\varepsilon}_{W}\right)$ and $d_{W}\left(\dot{\varepsilon}_{\mathrm{CB}}\right)$ comparable. A possible design of the second tag $\mathrm{MLA}_{\mathrm{CB}}$, with the purpose of maximizing the span of the two-port tag distance range, could be mainly constrained to achieve $d_{\mathrm{CB}}\left(\dot{\varepsilon}_{W}\right)<d_{W}\left(\dot{\varepsilon}_{W}\right)$, so extending the lower bound of $d$ toward zero.
The overall performances of the so obtained $\mathrm{MLA}_{\mathrm{CB}}$, placed $1.5 \mathrm{~cm}$ apart from the $\mathrm{MLA}_{W}$ and, hence, accounting for the interantenna coupling, are summarized in the second row of Fig. 3. The corresponding classification table (Fig. 4) indicates that the water target is recognized within the read distance $0.4 \mathrm{~m}<d<$ $2.2 \mathrm{~m}$, when the only ID associated with the $\mathrm{MLA}_{W}$ microchip $\left(I D_{W}\right)$ is collected by the reader. The chlorobenzene target, instead, can be sensed by $0.4 \mathrm{~m}<d<3.4 \mathrm{~m}$ when at least the $I D_{\mathrm{CB}}$ is received.

\section{EXPERIMENTAL EVALUATION}

A very preliminary laboratory experiment is discussed here. The purpose is to demonstrate the validity of the basic principle concerning the possibility to govern the variation of the two-port tag antenna features with respect to the change of a real tagged body. In particular, we have designed a two-MLA tag for the sensing of the filling level $h$ of a box. As introduced in Section II, the variation of the shape of the target modifies the apparent permittivity sensed by the antennas and, hence, all of their relevant parameters. With the aim to isolate and characterize the response of the antennas themselves, the experiment does not consider the RFID chip mounted on, nor the interrogation from a real reader. Moreover, readers that are able to measure the strength of the backscattered signals are up to now not so common on the market.

The target is a cubic box made of perspex $\left(\varepsilon_{r}=2.7, \sigma \simeq 0\right)$ having 5 - $\mathrm{mm}$ thickness and $20-\mathrm{cm}$ width. The box is filled up to a height $h$ (changed during the experiment) by sugar powder $\left(\varepsilon_{r}=3, \sigma \simeq 0\right)$. The box is placed over a large copper sheet ( 1 $\mathrm{m} \times 1 \mathrm{~m})$ acting as an image plane.

The two MLAs are intended to be placed vertically on the external side of the box. Due to the presence of the ground plane, monopole configurations are considered. Consequently, the impedance measurement is greatly simplified since no balun device is required. The antennas have regular turns $\left(w_{m k}=\right.$ $W_{1,2}, h_{m k}=H_{1,2}$, according to the convention in Fig. 6) and they are optimized for the best $\tau$ such that the MLA 1 and $\mathrm{MLA}_{2}$ are matched, at $870 \mathrm{MHz}$, to the microchip $\left(Z_{\text {chip }}=\right.$ $50-j 200 \Omega$ ) when the sugar level is $h=10 \mathrm{~cm}$ and $h=0$ cm (empty box), respectively. In this case, the design is performed by using a finite-difference time domain solver [45], accounting for the real box with all of the main geometrical and physical details. As in the previous numerical examples, the two MLAs are scaled replicas with $H_{1}\left(\mathrm{MLA}_{1}\right)=2.5 \mathrm{~cm}$, $W_{1}\left(\mathrm{MLA}_{1}\right)=1.1 \mathrm{~cm}$, and $H_{2}=1.1 H_{1}, W_{2}=1.1 W_{1}$. The overall antenna heights are $3.3 \mathrm{~cm}$ and $3.63 \mathrm{~cm}$, respectively. The distance between the MLAs' gaps is $4 \mathrm{~cm}$. The tag prototype is fabricated by 1 -mm-radius copper wire, and is shown in Fig. 15(a). The MLA monopoles are terminated on SMA connectors soldered on a $10 \times 6-\mathrm{cm}$ copper sheet which is then placed in front of the perspex box as indicated in Fig. 15(b). At this purpose, the large ground plane is properly drilled to accommodate the SMA connectors for the connection to the HP 8753C vector network analyzer by means of flexible coaxial cables. The $Z_{11}$ and $Z_{22}$ impedance of the tag are measured, having de-embedded the SMA connectors, when the filling level is increased in the range $0<h<10 \mathrm{~cm}$ with steps of $2 \mathrm{~cm}$. The measurements are repeated in the reverse order (by emptying the box) and the two resulting sets of data are finally averaged. 


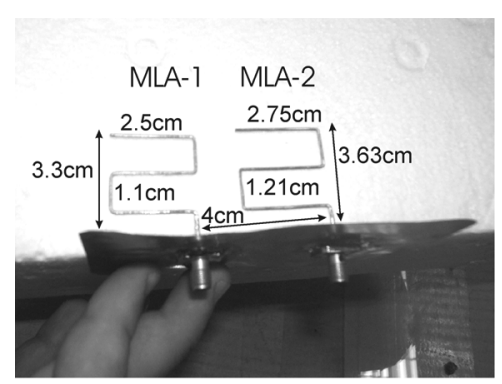

a)

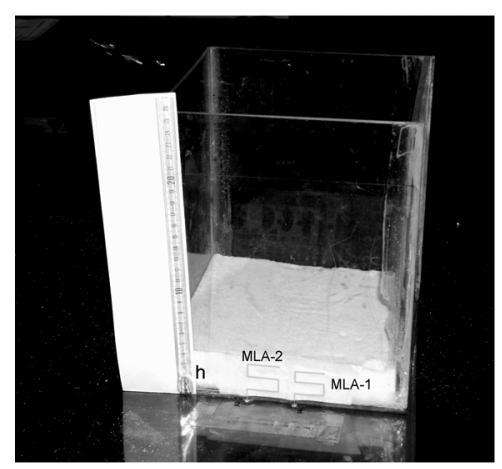

b)

Fig. 15. Powder level: experimental setup comprising the two MLA tags and a perspex cubic box of $20 \times 20$-cm cross-section partially filled with sugar up to a level $h$. Only half of the structure is considered since the copper ground plane acts as an image plane. The antennas are fixed to the box's vertical side by means of an adhesive ribbon.

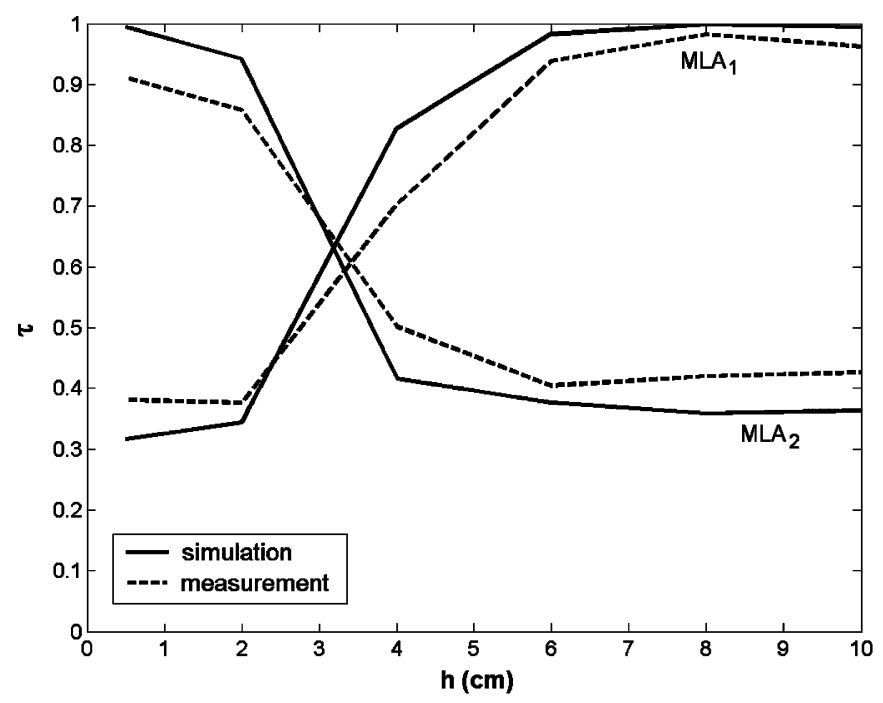

Fig. 16. Powder level: theoretical (simulated) and measured power transmission coefficients for the $\mathrm{MLA}_{1}$ optimized for $h=10 \mathrm{~cm}$, and $\mathrm{MLA}_{2}$, optimized for an empty box $(h=0)$.

The resulting matching diagram of the power transmission factor, estimated by FDTD and measured, is shown in Fig. 16. Beside the nice agreement between simulations and measurements, it is possible to appreciate that the $\tau$-curves are monotonic with the change of $h$ and that each port is rather mismatched in the condition for which the other one exhibits $\tau \rightarrow 1$.

The sensing ability of the two-port tag depends on the calibration curve $p_{21} \leftrightarrow h$, and on the ratio in (7). Our equipment only permits impedance measurements and, hence, we only consider

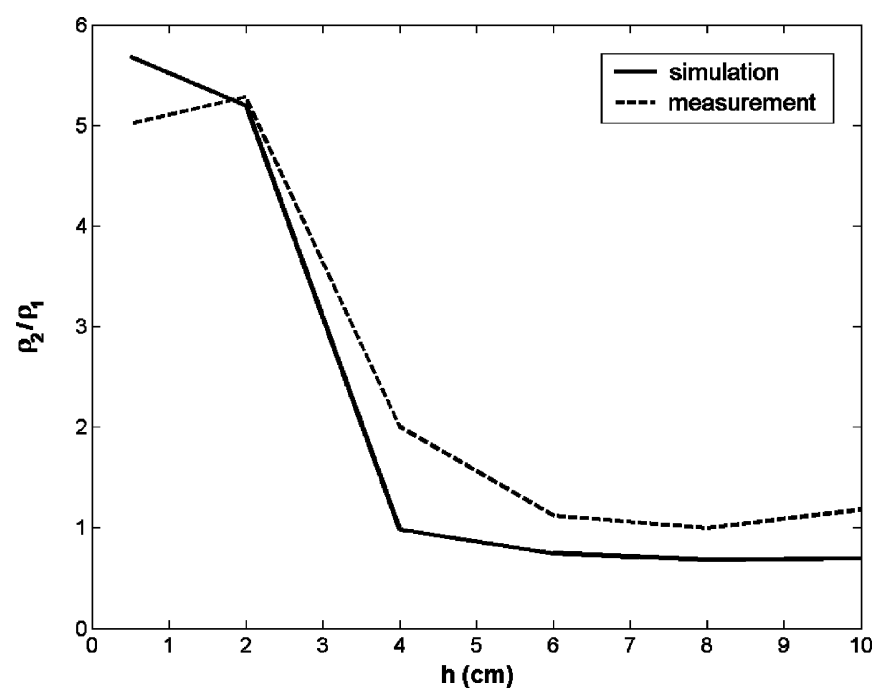

Fig. 17. Powder level: theoretical (simulated) and measured $\rho_{2} / \rho_{1}$ coefficient in (7) versus the sugar level $h$ inside the box.

the function $\rho_{2} / \rho_{1}[h]$, shown in Fig. 17. However, we preliminary evaluated, by numerical simulations, that the gain ratio $\left(G_{2} / G_{1}\right)^{2}$ is nearly unitary for the whole considered variation of the level $h$, at least for observation in front of the tag.

The behavior of the calibration curve is monotonic, except for a very early short part, and resembles that of the numerical example in Fig. 13 with a good dynamic $\left(1<p_{21}<5\right)$ when $2<h<8$. A saturation effect is clearly visible for levels higher than $h=8 \mathrm{~cm}$ (e.g., when the powder level greatly exceeds the vertical height of the antennas). In this condition, further increases in $h$ do not produce additional variation of the antenna responses, as shown in Fig. 16, and such a change of the target could not be therefore sensed since the sugar powder acts as an infinite medium for the two antennas. The sensed dynamic of the powder level could be increased by designing longer tags or using a vertical array of properly tuned tags.

\section{Discussions AND CONCLUSIONS}

A new idea has been presented to develop very low-cost passive RFID devices capable of monitoring the features of moving objects without the need for specific sensors.

The classification and the analog sensing potentiality of the multiport system have been formulated in a unitary framework, and some requirements on antenna design have been introduced, as deduced by the provided examples. The main assumption is that the radiation patterns referred to all of the ports are nearly proportional. It is worth noticing that the availability of at least two independent, but correlated baskscattered signals, which follow the same propagation rules inside the environment wherein the reader and tags are placed, makes this method potentially insensitive to the multipath induced by the interaction with the environment itself.

The application to a real class of targets demands a preliminary analysis of the permittivity range that has to be detected, or more, in general, of the range of the parameter to monitor. This will suggest how to select the reference permittivities and how to shape the antennas' layout so that the resulting S-RFID tag exhibits the required discrete or analog range, independent of the reader-tag orientation. 
TABLE IV

ClassiFicATION A TWO-PORT TAG

\begin{tabular}{|c|c|c|c|c|c|}
\hline $\begin{array}{l}\text { target } \\
\dot{\varepsilon}_{C B}\end{array}$ & $\begin{array}{c}\mathrm{ID}_{C B} \\
\mathrm{ID}_{W}\end{array}$ & $\begin{array}{l}\mathrm{ID}_{C B} \\
\mathrm{ID}_{W}\end{array}$ & $\begin{array}{l}\mathrm{ID}_{C B} \\
\mathrm{ID}_{W}\end{array}$ & $\mathrm{ID}_{C B}$ & l \\
\hline \multirow[t]{3}{*}{$\begin{array}{l}\text { target } \\
\dot{\varepsilon}_{W}\end{array}$} & $\begin{array}{c}\mathrm{ID}_{C B} \\
\mathrm{D}_{W}\end{array}$ & $\mathrm{ID}_{W}$ & 1 & / & I \\
\hline & \multicolumn{2}{|c|}{$\begin{array}{c}d_{C B}\left(\dot{\varepsilon}_{W}\right) \\
0.4 \mathrm{~m}\end{array}$} & \multicolumn{2}{|c|}{$\begin{array}{c}d_{W}\left(\dot{\varepsilon}_{C B}\right) \\
2.5 \mathrm{~m}\end{array}$} & \\
\hline & & & & $\begin{array}{r}d_{C B} \\
3 .\end{array}$ & ) \\
\hline
\end{tabular}

The considered basic $p_{i j} \leftrightarrow \varepsilon_{T}$ inversion technique is up to now mainly restricted to two-port tags (Table IV) and, thus, to a platform that is able to detect a single-parameter variation.

Some preliminary and simplified examples have demonstrated, by computer simulations and early laboratory experiments, the reasonableness of the proposed idea, especially for the case of low-loss targets. Nevertheless, further research efforts are required to develop a robust antenna-design methodology for both the cluster of tags and especially for the true multiport antenna wherein the same radiating body is shared by multiple chips. Moreover, the ultimate potentialities and limitation of the proposed sensing platform, customized for real applications, need to be verified by further prototypes and experimentation, including the microchips and the interaction with a reader. These important issues offer research materials for future dedicated papers.

The proposed platform could find application in many security and industrial contexts, for instance: 1) to monitor a (nonmetallic) container filled with low-loss liquids which could undergo changes along with the time; 2) to sense the filling percentage of a container; 3 ) to earn some preliminary information about the material filling a case without opening it or performing more detailed analysis; 4) to monitor the opening or tampering of a case also in consideration that the target history could be stored by the reader in the rewritable memory of the tag's microchip.

\section{ACKNOWLEDGMENT}

The authors would like to thank D. Scarana for technical support and E. Di Giampaolo for suggestions and valuable discussions. The measurements were performed at the Section of Toxicology and Biomedical Sciences of the ENEA Casaccia Research Centre, Rome, thanks to the valuable support of G. Lovisolo, R. Pinto, and S. Mancini. Patent Pending RM 2007 A 466.

\section{REFERENCES}

[1] I. F. Akyildiz, W. Su, Y. Sankarasubramaniam, and E. Cayirci, "A survey on sensor networks," IEEE Commun. Mag., vol. 40, no. 8, pp. 102-114, Aug. 2002.

[2] M. Aboelaze and F. Aloul, "Current and future trends in sensor networks: A survey," in Preoc. 2nd IFIP Int. Conf. Wireless Optical Communications Networks, Mar. 2005, pp. 551-555.

[3] M. A. M. Vieira, C. N. Coelho, Jr, D. C. da Silva, Jr, and J. M. da Mata, "Survey on wireless sensor network devices," in Proc. IEEE Conf. Emerging Technologies Factory Automation, Sep. 16-19, 2003, vol. 1 , pp. 537-544.

[4] T. Arampatzis, J. Lygeros, and S. Manesis, "A survey of applications of wireless sensors and wireless sensor networks," in Proc. 13th Mediterranean Conf. Control Automation, Cyprus, Turkey, Jun. 2005, pp. 719-724.
[5] R. Pon, A. Kansal, D. Liu, M. Rahimi, L. Shirachi, W. J. Kaiser, G. J. Pottie, M. Srivastava, G. Sukhatme, and D. Estrin, "Networked infomechanical systems (NIMS): Next generation sensor networks for environmental monitoring," in Proc. IEEE Microwave Symp. Digest, Jun. 12-17, 2005, pp. 373-376.

[6] J. Polastre, R. Szewczyk, A. Mainwaring, D. Culler, and J. Anderson, "Analysis of wireless sensor networks for habitat monitoring," in Proc. Int. Workshop Wireless Sensor Networks Applications, 2002, pp. 88-97.

[7] K. Lorincz, D. J. Malan, T. R. F. Fulford-Jones, A. Nawoj, A. Clavel, V. Shnayder, G. Mainland, M. Welsh, and S. Moulton, "Sensor networks for emergency response: Challenges and opportunities," IEEE Pervasive Comput. Mag., vol. 3, no. 4, pp. 16-23, Oct. 2004.

[8] B. Lo, "Key technical challenges and current implementations of body sensor networks," presented at the Int. Workshop on Wearable and Implantable Body Sensor Networks, London, U.K., Apr. 12-13, 2005, Imperial College.

[9] M. Philipose, K. P. Fishkin, M. Perkowitz, D. J. Patterson, D. Fox, H. Kautz, and D. Hahnel, "Inferring activities from interactions with objects," IEEE Pervasive Computing Mag., vol. 3, no. 4, pp. 50-57, Oct.-Dec. 2004.

[10] K. S. Low, W. N. N. Win, and M. J. Er, "Wireless sensor networks for industrial environments," in Proc. Int. Conf. Computational Intelligence for Modelling, Control Automation Int. Conf. Intelligent Agents, Web Technologies Internet Commerce, Nov. 28-30, 2005, vol. 2, pp. 271-276.

[11] T. He, S. Krishnamurthy, J. A. Stankovic, T. Abdelzaher, L. Luo, R. Stoleru, T. Yan, L. Gu, J. Hui, and B. Krogh, "Energy-efficient surveillance system using wireless sensor networks," in Proc. Int. Conf. Mobile Systems, Applications Services, Boston, MA, 2004, pp. 270-283.

[12] T. He, S. Krishnamurthy, J. A. Stankovic, T. Abdelzaher, L. Luo, R Stoleru, T. Yan, L. Gu, J. Hui, and B. Krogh, "Vigilnet: An integrated sensor network system for energy-efficient surveillance," ACM Trans. Sensor Netw., vol. 2, no. 1, pp. 1-38, Feb. 2006.

[13] D. J. Cook and S. K. Das, "How smart are our environments? An updated look at the state of the art," Pervasive Mobile Comput., vol. 3, no. 2, pp. 53-73, Mar. 2007.

[14] M. Srivastava, R. Muntz, and M. Potkonjak, "Smart kindergarten: Sensor-based wireless networks for smart developmental problem-solving enviroments," in Proc. 7th Annu. Int. Conf. Mobile Comput. Networking, Rome, Italy, 2001, pp. 132-138.

[15] B. A. Warneke and K. S. J. Pister, "MEMS for distributed wireless sensor networks," in Proc. 9th Int. Conf. Electronics, Circuits Systems, 2002, vol. 1, pp. 291-294.

[16] A. Lecointre, D. Dragomirescu, D. Dubuc, K. Grenier, P. Pons, A. Aubert, A. Muller, P. Berthou, T. Gayraud, and R. Plana, "Miniaturized wireless sensor networks," in Proc. Int. Semiconductor Conf., Sep. 2006, vol. 1, pp. 13-17.

[17] L. Wang and Y. Xiao, "Energy saving mechanisms in sensor networks," in Proc. 2nd Int. Conf. Broadband Networks, Oct. 3-7, 2005, vol. 1, pp. 724-732.

[18] A. D. Joseph, "Energy harvesting projects," IEEE Pervasive Comput. Mag., vol. 4, no. 1, pp. 69-71, Jan.-Mar. 2005.

[19] A. Kansal and M. B. Srivastava, "An environmental energy harvesting framework for sensor networks," in Proc. Int. Symp. Low Power Electronics Design, Seoul, Korea, 2003, pp. 481-486.

[20] S. Nambi, S. Nyalamadugu, S. M. Wentworth, and B. A. Chin, "Radio frequency identification sensors," in Proc. 7th World Multiconf. Systemics, Cybernetics Informatics, 2003, pp. 386-390.

[21] C. Alippi and G. Vanini, "An application-level methodology to guide the design of intelligent-processing power-aware passive RFID," in Proc. Int. Symp. Circuits System, 2005, vol. 6, pp. 5509-5512.

[22] M. Philipose, J. Smith, B. Jiang, A. Mamishev, S. Roy, and K. Sundara-Rajan, "Battery-free wireless identification and sensing," IEEE Pervasive Comput. Mag., vol. 4, no. 1, pp. 10-18, Jan.-Mar. 2005.

[23] G. Marrocco, "RFID antennas for the UHF remote monitoring of human subjects," IEEE Trans. Antennas Propag., vol. 55, no. 6, pt. 2, pp. 1862-1680, Jun. 2007.

[24] J. R. Smith, B. Jiang, S. Roy, M. Philipose, K. Sundara-Rajan, and A. Mamishev, "ID modulation: Embedding sensor data in an RFID timeseries," presented at the 7th Information Hiding Workshop, Barcelona, Spain, Jun. 6-8, 2005.

[25] A. P. Sample, D. J. Yeager, P. S. Powledge, and J. R. Smith, "Design of a passively-powered, programmable sensing platform for UHF RFID systems," in Proc. IEEE Int. Conf. RFID, Grapevine, TX, Mar. 26-28, 2007, pp. 149-156.

[26] L. M. Reindl, A. Pohl, G. Scholl, and R. Weigel, "SAW-based radio sensor systems," IEEE Sensors J., vol. 1, no. 1, pp. 69-77, Jun. 2001. 
[27] L. Reindl, G. Scholl, T. Ostertag, H. Scherr, U. Wolff, and F. Schmidt, "Theory and application of passive SAW radio transponders as sensors," IEEE Trans. Ultrason., Ferroelectr. Freq. Control, vol. 45, no. 5, pp. 1281-1292, Sep. 1998.

[28] IEEE Standard Definitions of Terms for Antennas, IEEE Std. 145-1993, 1993.

[29] P. V. Nikitin and K. V. S. Rao, "Theory and measurement of backscattering from RFID tags," IEEE Antennas Propag. Mag., vol. 48, no. 6, pp. 212-218, Dec. 2006.

[30] C. A. Balanis, Antenna Theory: Analysis and Design, 2nd ed. New York: Wiley, 1997.

[31] E. F. Knott, M. T. Tuley, and J. F. Shaeffer, Radar Cross Section, 2nd ed. Raleigh, NC: SciTech, 2004.

[32] P. Nikitin and K. V. S. Rao, "Performances limitations of passive UHF RFID systems," in Proc. IEEE Antennas Propag. Soc. Int. Symp., 2005, pp. 1011-1014.

[33] C. Polk, "Resonance and supergain effects in small ferromagnetically or dielectrically loaded biconical antennas," IEEE Trans. Antennas Propag., vol. AP-7, no. 5, pp. 414-423, Dec. 1959.

[34] R. W. P. King and G. S. Smith, Antennas in Matter. Cambridge, MA: MIT Press, 1981.

[35] R. E. Collin, Foundation for Microwave Engineering. New York: McGraw-Hill, 1992, pp. 160-160.

[36] J. R. Smith, K. P. Fishkin, B. Jiang, A. Mamishev, M. Philipose, A. D. Rea, S. Roy, and K. Sundara-Rajan, "RFID-based techniques for human activity recognition," Commun. ACM, vol. 48, no. 9, pp. 39-44, Sep. 2005.

[37] C. Balanis, Advanced Engineering Electromagnetics. New York: Wiley, 1989, pp. 73-80.

[38] R. Garg, P. Bhartia, I. Bahl, and A. Ittipiboon, Microstrip Antenna Design Handbook. Boston, MA: Artech House, 2001, pp. 279-285.

[39] G. Marrocco, "Gain-optimized self-resonant meander line antennas for RFID applications," IEEE Antennas Wireless Propag. Lett., vol. 2, pp. 302-305, 2003.

[40] Y. Rahmat-Samii and E. Michielsen, Electromagnetic Optimization by Genetic Algorithms. New York: Wiley, 1999.

[41] “FEKO User's Manual, Suite 5.1," EM Software \& Systems-S.A. (Pty) Ltd., Stellenbosch, South Africa, 2005. [Online]. Available: http://www.feko.info.

[42] N. Miura, S. Yagihara, and S. Mashimo, "Microwave dielectric properties of solid and liquid foods investigated by time-domain reflectometry," J. Food Sci., vol. 68, no. 4, pp. 1396-1403, 2003.

[43] "Regulatory status for using RFID in the UHF spectrum." [Online]. Available: http://www.epcglobalinc.org/tech/freq\_reg/RFID\_at \_UHF\_Regulations \20070504.pdf.

[44] K. Fenske and D. Misra, "Dielectric materials at microwave frequencies," Appl. Microw. Wireless, vol. 12, no. 10, pp. 92-100, Oct. 2000.

[45] G. Marrocco and F. Bardati, "Best: A finite-difference solver fo time electromagnetics," Simulation Practice Theory, no. 7, pp. 279-293, 1999.

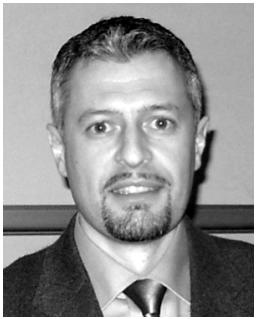

Gaetano Marrocco (M'98) was born in Teramo, Italy, on August 29, 1969. He received the Laurea degree in electronic engineering and the Ph.D. degree in applied electromagnetics from the University of L'Aquila, Italy, in 1994 and 1998, respectively.

He has been a Researcher at the University of Rome "Tor Vergata," Rome, since 1997, where he currently teaches Antenna Design and Bioelectromagnetics. In 1994, he was a Postgraduate Student at the University of Illinois at Urbana Champain. In 1999, he was a Visiting Scientist at the Imperial College, London, U.K. His research is mainly devoted to the modelling and design of broadband and ultra-wideband antennas and arrays as well as miniaturized antennas for RFID applications. He has been involved in several space, avionic, naval, and vehicular programs of the European Space Agency, NATO, Italian Space Agency, and the Italian Navy. He holds two patents on broadband naval antennas and one patent on sensor RFID systems. He is currently Associate Editor of the IEEE ANTENNAS AND WIRELESS PROPAGATION LETTERS.

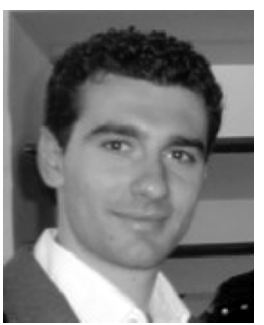

Lorenzo Mattioni was born in Rome, Italy, on January 8, 1979. He received the Laurea degree in telecommunications engineering and the Ph.D. degree in electromagnetics from the University of Rome "Tor Vergata," Rome, in 2004 and 2008, respectively.

His main scientific interests are the modeling and design of innovative broadband antenna systems for mobile platforms and for the software- defined radio technology. Other scientific interests include the design of compact multiband antennas for mobile devices, the modeling and analysis of colocated antenna systems in complex electromagnetic environments, and the design of miniaturized/low-profile antennas for RFID systems. He is the coauthor of two international patents on broadband multifunction naval antennas.

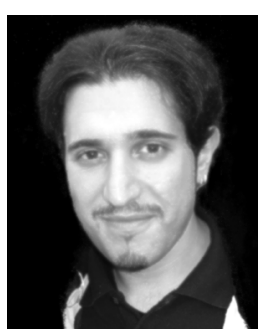

Claudio Calabrese was born in Rome, Italy, on November 15, 1982. He received the Laurea degree in telecommunications engineering from the University of Rome "Tor Vergata," Rome, in 2007.

His main scientific activity concerns small antenna design and electromagnetic optimization. He is currently working on design, experimental assessment, and maintenance of radio base stations for indoor and outdoor cellular coverage at Telind S.r.1, Rome. 